\title{
Comaximal factorization in a commutative Bezout ring
}

\section{B. Zabavsky, O. Romaniv, B. Kuznitska, and T. Hlova}

\author{
Communicated by E. Zelmanov
}

A BSTRACT. We study an analogue of unique factorization rings in the case of an elementary divisor domain.

One of the main sources for all researches in the present paper is an elementary divisor ring. Recall that a matrix over an associative ring with identity has a canonical diagonal reduction if it can be reduced to a diagonal form by left and right multiplications by some invertible matrices so that each diagonal element is a total divisor of the subsequent one. If any matrix over an associative ring with identity has a canonical reduction then such a ring is called an elementary divisor ring [1].

Most of the known classes of elementary divisor rings significantly depend on ascending chain condition on ideals. The first example of a classical elementary divisor ring without ACC on ideals was found by Vanderbern in 1915, namely, the ring of analytic functions [2]. In a more abstract form, this example allowed Helmer [3] to introduce a new class of elementary divisor rings called the class of adequate rings.

All notations and necessary definitions are well-known. They can also be found in [5].

Recall, that an element $a$ of a commutative $\operatorname{ring} R$ with identity is said to be adequate, if for any element $b \in R$ one can find elements $r, s \in R$ such that the decomposition $a=r \cdot s$ satisfies the following properties:

2010 MSC: 13F15, 19B10.

Key words and phrases: Bezout ring, clean ring, neat ring, elementary divisor ring, stable range one, stable range two, neat range one, pseudo-irreducible element. 
1) $r R+b R=R$,

2) $s^{\prime} R+b R \neq R$ for any noninvertible divisor $s^{\prime}$ of element $s$.

If any nonzero element of a ring $R$ is an adequate element, then $R$ is called an adequate ring.

Henriksen observed that in an adequate ring each nonzero prime ideal is contained in a unique maximal ideal [4]. Furthermore Henriksen showed that there exist elementary divisor rings which are not adequate [4].

A specific role in modern research of elementary divisor rings is played by a K-theoretical invariant such as stable range. For example, an important role in studying of the elementary divisor rings is played by the Hermite rings. A ring is called a right (left) Hermite ring if any $1 \times 2$ $(2 \times 1)$ matrix over this ring has diagonal reduction over this ring. An Hermite ring is a ring which is both right and left Hermite.

Note that any Hermite ring is a finitely generated principal ideal ring (in what follows we will call it a Bezout ring), i.e. a ring in which any finitely generated left or right ideal is principal. In the case of commutative rings we have the following result.

Theorem 1 ([5]). A commutative Bezout ring is an Hermite ring if and only if it is a ring of stable range 2.

We say that a ring $R$ has stable range 2 if for any elements $a, b, c \in R$ the equality $a R+b R+c R=R$ implies that there are some elements $\lambda, \mu \in R$ such that $(a+c \lambda) R+(b+c \mu) R=R$.

In the class of commutative Bezout rings of stable range 2 we have that a Bezout ring is an elementary divisor ring if and only if it is a ring of neat range 1 .

Theorem 2 ([6]). A commutative Bezout domain is an elementary divisor ring if and only if it is a ring of neat range 1 .

A commutative ring $R$ is said to be of neat range 1 if for any $a, b \in R$ such that $a R+b R=R$ there exists $t \in R$ such that for the element $a+b t$ the ring $R /(a+b t) R$ is a clean ring.

A ring $R$ is called clean if each element of $R$ is the sum of the unit and an idempotent. Recall, that an element $a$ of a commutative ring $R$ is called neat if $R / a R$ is a clean ring. Notice that an indecomposable ring is clean if and only if it is local.

Proposition 1. Let $R$ be a commutative Bezout ring and a be a nonzero and nonunit element $R$ such that $R / a R$ is an indecomposable ring. Then for any representation $a=r \cdot s$, where $r \notin U(R), s \notin U(R)$ we have $r R+s R \neq R$. 
Proof. Let $\bar{R}=R / a R$ be an indecomposable ring. Suppose that $a=r \cdot s$, where $r \notin U(R), s \notin U(R)$, we have $r R+s R=R$. Then $r u+s v=1$ for some elements $u, v \in R$. Let $\bar{r}=r+a R, \bar{s}=s+a R, \bar{u}=u+a R$ and $\bar{v}=v+a R$, then $\bar{r}^{2} \bar{u}=\bar{r}$ and $\bar{s}^{2} \bar{v}=\bar{s}$. Since $\bar{R}$ is indecomposable and $\overline{r u}^{2}=\overline{r u}$, we have $\overline{r u}=\overline{0}$ or $\overline{r u}=\overline{1}$. If $\overline{r u}=\overline{0}$ then $\overline{s v}=\overline{1}$, because $\overline{r u}+\overline{s v}=\overline{1}$, namely $s R+a R=R$.

Since $a=r \cdot s$, it is possible only if $s \in U(R)$. This contradicts the selection of an element $s$. If $\overline{r u}=\overline{1}$ then $\overline{s v}=\overline{0}$, and similarly we have $r \in U(R)$. However, this is not possible.

Proposition 2. Let a be a nonzero nonunit element of a commutative ring $R$. Then $R / a R$ is an indecomposable ring if and only if for any element $b$ such that $b(1-b) \in a R$ we have $b \in a R$ or $1-b \in a R$.

Proof. Suppose that for any element $b$ such that $b(1-b) \in a R$ we have $b \in a R$ or $1-b \in a R$. Let $\bar{R}=R / a R$ and $\bar{e}$ be an idempotent of $\bar{R}$. Let $\bar{e}=e+a R$. Since $\bar{e}^{2}=\bar{e}$, this implies $e(1-e) \in a R$. Then $e \in a R$ or $1-e \in a R$, i.e. $\bar{e}=\overline{0}$ or $\bar{e}=\overline{1}$.

Conversely, suppose that $\bar{R}=R / a R$ is an indecomposable ring and there are elements $r$ and $s$ with $r R+s R=R$, and if $r \cdot s \in a R$ then $r \notin a R$ and $s \notin a R$. Write $r s=a t$ and, as $r R+s R=R$, we have $r x+s y=1$ for some elements $x, y \in R$. Then

$$
r x=r^{2} x^{2}+r x s y=r^{2} x^{2}+a t x y
$$

and

$$
r x(1-r x)=a t x y .
$$

Let $\overline{r x}=r x+a R$. Thus $\overline{r x}$ is an idempotent of $\bar{R}$. We claim that $\overline{r x}$ is a nontrivial idempotent. If $\overline{r x}=\overline{0}$ then $r x \in a R$. Since $r x+s y=1$,

$$
r^{2} x+r s y=r^{2} x+a t y=r r x+a t y \in a R .
$$

Since $r^{2} x+r s y=r(r x+s y)=r$, we have $r \in a R$, which is a contradiction. If $\overline{r x}=\overline{1}$ we have $1-r x \in a R$, i.e. $s y \in a R$. Since $r x+s y=1$, then $r s x+s^{2} y=s$ and we have $s \in a R$, which is again a contradiction.

Proposition 3. Let a be a nonzero nonunit element of a commutative ring $R$ such that $R / a R$ is an indecomposable ring. Then for any representation $a=b c$, where $b R+c R=R$, either $b \in U(R)$ or $c \in U(R)$. 
Proof. Let $\bar{R}=R / a R$ be an indecomposable ring. Suppose that $a=b c$, where $b \notin U(R), c \notin U(R)$ and $b R+c R=R$ for some elements $b, c \in R$. Let $b u+c v=1$ for some elements $u, v \in R$. Then $\bar{b}^{2} \bar{u}=\bar{b}$, where $\bar{b}=b+a R$, $\bar{u}=u+a R$. Since $R$ is an indecomposable ring and $(\bar{b} \bar{u})^{2}=\bar{b} \bar{u}$, we have $\bar{b} \bar{u}=\overline{0}$ or $\bar{b} \bar{u}=\overline{1}$. If $\bar{b} \bar{u}=\overline{0}$, then $b u=a t$ for some element $t \in R$. Since $b u+c v=1$ and $b u=a t$ we have $a t+c v=c(b t+v)=1$, i.e. $c \in U(R)$, but this is impossible. If $\bar{b} \bar{u}=\overline{1}$ then $b u-1=a s$ for some element $s \in R$. Then

$$
b u-a s=b u+b c s=b(u+c s)=1,
$$

i.e. $b \in U(R)$, and this is impossible.

Definition 1. Let $R$ be a commutative ring. An element $a \in R$ is called pseudo-irreducible if for any representation $a=b c$, where $b R+c R=R$, we have $b \in U(R)$ or $c \in U(R)$.

Definition 2. Let $R$ be a commutative ring. An element $a \in R$ is called pseudo-prime if for any elements $b, c \in R$ such that $b c \in a R$ and $b R+c R=R$ we have $b \in a R$ or $c \in a R$.

By Proposition 3 we have the following results.

Proposition 4. For a commutative ring any pseudo-prime element is pseudo-irreducible.

Proposition 5. Let $R$ be a commutative Bezout domain. Then any pseudo-irreducible element $R$ is pseudo-prime.

Proof. Let $a$ be a pseudo-irreducible element of $R$. Let $b c \in a R$ and $b R+c R=R$. Consider $b R+a R=d R$, then $b=d b_{0}, a=d a_{0}$ and $b_{0} R+a_{0} R=R$. Let $b_{0} u+a_{0} v=1$ for some elements $u, v \in R$. Since $b, c \in a R$, then $b c=a t$ for some element $t \in R$. Consider $b, c=d b_{0} c=d a_{0} t$, then $b_{0} c=a_{0} t$. Since $b_{0} u+a_{0} v=1$, then

$$
c=b_{0} c u+a_{0} c v=a_{0} t u+a_{0} c v=a_{0}(t u+c v) .
$$

Since $b R+c R=R$ and $c \in a_{0} R$, we have $a_{0} R+d R=R$. Since $a$ is a pseudo-irreducible element and $a=a_{0} d$, where $a_{0} R+d R=R$, we have $a_{0} \in U(R)$ or $d \in U(R)$. If $a_{0} \in U(R)$ then $b \in a R$. If $d \in U(R)$ then $c \in a R$.

Clearly, every nonunit and nonzero element in a local ring is a pseudoirreducible element. 
An element $a$ of a commutative ring $R$ is said to be colocal if $R / a R$ is a local ring. Obviously, any atom and any power of an atom is a colocal element.

By Proposition 5 and the fact that any local ring is an indecomposable ring, we will notice that a colocal element is a pseudo-irreducible element.

Note that any clean element is a neat element [7]. Since any local ring is a clean ring, we have that any colocal element is a neat element. Note that a commutative clean ring is indecomposable if and only if it is a local ring.

The following fact will be useful to us.

Proposition 6. Any nonunit divisor of a neat element of a commutative Bezout domain is a neat element.

Proof. Let $R$ be a commutative Bezout domain and let $a$ be a neat element of $R$. By [6], for every elements $b, c \in R$ such that $b R+c R=R$ there exists $a \in R$ such that $a=r \cdot s$, where $r R+b R=R, s R+c R=R$ and $r R+s R=R$.

Let $a=x \cdot y$, then $r R+x R=\alpha R, r=\alpha r_{0}, x=\alpha x_{0}$ for some elements $r_{0}, x_{0} \in R$ such that $r_{0} R+x_{0} R=R$. Since $a=r \cdot s=x \cdot y$, we have $\alpha r_{0} s=\alpha x_{0} y$. Then $r_{0} s=x_{0} y$. Since $r_{0} R+x_{0} R=R$, we have $r_{0} u+x_{0} v=1$ for some elements $u, v \in R$. Since $r_{0} s=x_{0} y$, we have $r_{0} s u+x_{0} s v=s, x_{0}(y u+s v)=s$. We have $x=\alpha x_{0}$, where $\alpha R \in b R$, $x_{0} R \in c R$ and $\alpha R+x_{0} R=R$, as $r R \subset \alpha R$ and $s R \subset x_{0} R$, i.e. $x$ is a neat element.

By Proposition 4 we have the following result.

Proposition 7. A neat element of a commutative ring is pseudo-prime if and only if it is a colocal element.

Proposition 8. Any colocal element of a commutative ring $R$ is an adequate element.

Proof. Let $a$ be a colocal element. Since $R / a R$ is a local ring, there exist a unique maximal ideal $M$ such that $a \in M$. For any element $b \in R$ we have two cases:

1) $b \in M$, then $a=1 \cdot a$, where $1=r$ and $s=a$ and for each nonunit divisor $s^{\prime}$ of $a$ we have $s^{\prime} R+b R \subset M$, i.e. $s^{\prime} R+b R \neq R$;

2) $b \notin M$, then we have $a R+b R=R$. 
Definition 3. Let $R$ be a commutative ring and $a$ be a nonzero, nonunit element of $R$. We will call $a=a_{1} \cdot a_{2} \cdots a_{n}$ a complete comaximal factorization of $a$ if the $a_{i}$ are pairwise comaximal pseudo-irreducible elements.

We will call $R$ a comaximal factorization ring if any nonzero nonunit element of $R$ has a complete comaximal factorization.

By [6], any adequate element of a commutative Bezout ring is a neat element. In the case of element with complete comaximal factorization, we have the following result.

Theorem 3. Let $R$ be a commutative Bezout domain. If a is a neat element with complete comaximal factorization, then $a$ is an adequate element.

Proof. Let $a=a_{1} \cdot a_{2} \cdots a_{n}$ be a complete comaximal factorization of $a$, where $a_{i}$ are pairwise comaximal pseudo-irreducible elements. Since $a$ is a neat element, by Proposition 6, we have that $a_{i}$ is a neat element. Moreover, by Proposition 7, we have that $a_{i}$ is a colocal element. By Proposition $8, a_{i}$ is an adequate element. Since the product of adequate elements is an adequate element [5], we obtain that $a$ is an adequate element.

Definition 4. Let $R$ be a commutative ring. An element $a \in R$ is said to be an element of stable range 1 if for any element $b \in R$ such that $a R+b R=R$ we have $(a+b t) R=R$ for some element $t \in R$.

Recall that a ring $R$ is said to be a ring of stable range 1 if for any elements $a, b \in R$ such that $a R+b R=R$ we have $(a+b t) R=R$ for some element $t \in R$.

Any colocal element is an example of a nontrivial element of stable range 1 . We notice that any idempotent of a commutative ring is an element of stable range 1 . Indeed, let $R$ be a commutative ring and let $e^{2}=e \in R$ and $e R+b R=R$ for $b \in R$. Then $e u+b v=1$ for some elements $u, v \in R$ and $(1-e) e u+(1-e) b v=1-e$. Then $(1-e) b v=1-e$, i.e. $e+b(1-e) v=1$. Because of the arbitrariness of an element $b$ we have that $e$ is an element of stable range 1 . Note that the product of the elements of stable range 1 is an elements of stable range 1 . We obtain the following result.

Theorem 4. Let $R$ be a commutative Bezout domain. Any neat element with complete comaximal factorization is an element of stable range 1. 
Proof. Let $a=a_{1} \cdot a_{2} \cdots a_{n}$, where $a_{i}$ are pairwise comaximal pseudoirreducible elements. Since $a$ is a neat element, we have that $a_{i}$ are colocal elements. Since $a_{i}$ are elements of stable range 1, then $a$ is an element of stable range 1.

An example of a commutative comaximal factorization Bezout domain can be found using this result.

Theorem 5. Let $R$ be a commutative ring. Each nonzero nonunit element of $R$ which has only finitely many prime ideals minimal over it has complete comaximal factorization.

Proof. Let's prove by induction on the power of the set of minimal prime ideals over elements. Let $a$ be an element with unique minimal prime ideal $P$ over it.

Suppose the contrary, i.e. $a=b \cdot c$, where $b R+c R=R$ and either $b \notin U(R)$ or $c \notin U(R)$. Since $a=b \cdot c \in P$ and $b R+c R=R$ then $b \in P$ and $c \notin P$ (or $b \notin P$ and $c \notin P$ ). Since $c R \neq R$, there exists a prime ideal $N$ such that $c \in N$. Since $a R \subset c R$, we have $a \in N$. Since $P$ is a unique minimal prime ideal over $a$, we have $P \subset N$. Since $b \in P$ and $c \in N$, then $R=b R+c R \subset N$ which is impossible, i.e. $a$ is a pseudo-irreducible element. Assume that the statement is proved for all elements such that the power of the set of minimal prime ideals over them is equal to $n$.

Let $a$ be an element of $R$ for which the power of the set of minimal prime ideals over it is equal to $n+1$. If $a$ is a pseudo-irreducible element then everything is proved.

Let $a=b \cdot c$, where $b R+c R=R$ and $b \notin U(R)$ or $c \notin U(R)$. Then the comaximality of $b$ and $c$ follows that the set of minimal prime ideals over $a$ is the disjoint union of the set of minimal prime ideals over $b$ and the set of minimal prime ideals over $c$. As $b$ and $c$ are nonunits, both these sets are nonempty, thus both are proper subsets of the set of minimal prime ideals over $a$, and hence are both of smaller power then the power set of the set minimal prime ideals over $a$.

Therefore both $b$ and $c$ have complete comaximal factorization, and then $a$ have complete comaximal factorization.

For a commutative ring $R$, the Zariski topology on $\operatorname{Spec} R$ is the topology obtained by taking the collection of sets of the form $U(I)=$ $\{P \in \operatorname{Spec} R \mid I \nsubseteq P\}$ (resp. $V(I)=\{P \in \operatorname{Spec} R \mid I \subseteq P\}$ ), for every ideal $I$ of $R$, as the open (resp. the closed) sets. When considered as a subspace of $\operatorname{Spec} R, \operatorname{Max}(R)$ is called the max-spectrum of $R$. Thus, its open and 
closed subsets are $U_{R}(I)=U(I) \cap \operatorname{Max}(R)=\{M \in \operatorname{Max}(R) \mid I \subseteq M\}$ and $V_{R}(I)=V(I) \cap \operatorname{Max}(R)=\{M \in \operatorname{Max}(R) \mid I \subseteq M\}$, respectively.

A topological space $X$ is called Noetherian if every nonempty set of closed subsets of $X$, ordered by inclusion, has a minimal element. An ideal $I$ of $R$ is called a $J$-radical ideal, if it is the intersection of all maximal ideals containing it. Clearly, $J$-radical ideals of $R$ correspond to closed subsets of $\operatorname{Max}(R)$. Recall that a ring $R$ is called $J$-Noetherian if it satisfies the ascending chain condition on $J$-radical ideals. For a commutative Bezout domain $R$, the $J$-Noetherian condition is equivalent to the condition that every nonzero nonunit element of $R$ has only finitely many prime ideals minimal over it.

By [8], we have the following result.

Theorem 6. A commutative Bezout domain is comaximal factorization if and only if $R$ is a $J$-Noetherian ring.

Definition 5. A commutative domain $R$ has finite character if each nonzero element of $R$ is contained in at most finitely many maximal ideals of $R$.

A commutative domain $R$ is $h$-local if $R$ has finite character and each nonzero prime ideal of $R$ is contained in a unique maximal ideal.

Consequently we have the following result.

Theorem 7. Let $R$ be a commutative Bezout domain whose each nonzero prime ideal is contained in a unique maximal ideal. Then $R$ is a comaximal factorization if and only if $R$ is an h-local domain.

Proof. If $R$ is a comaximal factorization domain then by Theorem 6 for each nonzero element $a$ of $R$ there is an element with only finitely many prime ideals minimal over it. Since each nonzero prime ideal $R$ is contained in a unique maximal ideal, we have that $a$ is contained in at most finitely many maximal ideals of $R$, i. e. $R$ has finite character.

If $R$ is an $h$-local Bezout domain then any nonzero nonunit element has only finitely many prime ideals minimal over it. By Theorem $5, R$ is a ring with complete comaximal factorization.

Since any commutative $h$-local Bezout domain is an adequate domain [9] we obtain the following results.

Theorem 8. Let $R$ be a commutative Bezout domain in which each nonzero prime ideal is contained in a unique maximal ideal and $R$ be a comaximal factorization ring. Then $R$ is an adequate domain. 
Since any commutative adequate Bezout domain is a ring in which each nonzero prime ideal is contained in a unique maximal ideal, we obtain the following results.

Theorem 9. Commutative adequate Bezout domain $R$ is comaximal factorization if and only if $R$ is a h-local Bezout domain.

Note that a commutative Bezout ring of stable range 1 is an elementary divisor ring. In the sequel we will study a commutative ring of stable range 2 which is not a ring of stable range 1 .

If $R$ is an elementary divisor ring which is not of stable range 1, then by Theorem $2, R$ contains a nonunit neat element. If in $R$ any neat element has a complete comaximal factorization then, by Proposition 7 and Proposition 8, any neat element is an adequate element, i.e. we obtain the following result.

Theorem 10. Let $R$ be a commutative elementary divisor domain which is not a ring of stable range 1 and any neat element of $R$ has a complete comaximal factorization. Then there exists a nonunit adequate element in $R$.

In particular, as a consequence we obtain the following result.

Theorem 11. For a commutative $J$-Noetherian Bezout domain $R$ we have:

$R$ is a ring of stable range 1 or $R$ contains a nonunit adequate element which is an element of stable range 1 .

Proof. If $R$ is a $J$-Noetherian Bezout domain which is not of stable range 1 , then by Theorem 2, $R$ has a neat element which has a complete comaximal factorization $a=a_{1} \cdot a_{2} \cdots a_{n}$, where $a_{i}$ are pairwise comaximal pseudoirreducible elements. By Proposition 6 and Proposition 7, $a_{i}$ are colocal elements. By Propositions 7 and $8, a_{i}$ are adequate elements of stable range 1 and $a$ is an adequate element of stable range 1 .

We recall the transfinite definition of the Krull dimension $K \operatorname{dim} M$ of a module $M$ (see [10]). By definition, we assume that zero modules have Krull dimension equal to -1 , and every nonzero Artinian module has Krull dimension equal to zero. Let us assume that $\alpha>0$ is an ordinal of the modules with Krull dimension $\beta$ are defined for all ordinal numbers $\beta<\alpha$ and $M$ is a module such that $K \operatorname{dim} M \neq \beta$. One says that the Krull dimension $K \operatorname{dim} M$ is equal to $\alpha$ if for any infinite properly descending 
chain $M_{1}>M_{2}>\ldots$ of submodules in $M$, there exists a positive integer $n$ such that $K \operatorname{dim} M_{n} / M_{n+1}<\alpha$.

All factor modules and submodules of a module with Krull dimension have Krull dimension. For a ring $R$ the right Krull dimension $K \operatorname{dim} R_{R}$ is the Krull dimension of the module $R_{R}$, if it exists.

A module $M$ is said to be finite-dimensional if $M$ does not contain a direct sum of infinite number of nonzero submodules. Each module which has Krull dimension is finite-dimensional [10].

Moreover, we have the following result.

Theorem 12. Let $R$ be a commutative Bezout ring. The following conditions are equivalent:

1) $R$ has Krull dimension.

2) Every factor ring of the ring $R$ is finite-dimensional and does not have proper idempotent essential ideals.

A submodule $W$ is a waist if every submodule either contains $W$ or is contained in $W$.

We have the following result.

Proposition 9. A finite-dimensional commutative and indecomposable Bezout ring $R$ has a unique minimal prime ideal which is a waist in $R$.

Let $R$ be a commutative Bezout domain which has Krull dimension.

By [11], $R$ is an elementary divisor ring. According to Theorem $2, R$ is a ring of neat range 1 . If $R$ is not a ring of stable range 1 then $R$ has no unit neat element.

Let $a$ be a pseudo-irreducible element of $R$. Then $R / a R$ is a finitedimensional indecomposable Bezout ring. By Proposition $9, \bar{R}=R / a R$, which has a unique minimal ideal $\operatorname{rad} \bar{R}$, which is a waist in $\bar{R}$. Since idempotents lift modulo a nil ideal, $\bar{R} / \operatorname{rad} \bar{R}$ is an indecomposable Bezout ring, i.e. $\bar{R} / \operatorname{rad} \bar{R}$ is a domain.

Thus, we proved that if $R$ is a commutative Bezout domain which has Krull dimension, then any pseudo-irreducible element $a \in R$ is an element such that there exists a prime ideal $P$ such that for any prime ideals which contain $a$ either $P \subset N$ or $N \subset P$.

\section{References}

[1] I. Kaplansky, Elementary divisor ring and modules, Trans. Amer. Math. Soc., v.66, 1949, pp.464-491.

[2] J. H. M. Wedderburn, On matrices whose coefficients are functions of single variable, Trans. Amer. Math. Soc., v.16, N.2, 1915, pp.328-332. 
[3] O. Helmer, The elementary divisor theorem for certain rings without chain conditions, Bull. Amer. Math. Soc., V.49, N.2, 1943, pp.225-236.

[4] M. Henriksen, Some remarks about elementary divsor rings, Michigan Math. J., v.3, 1955-1956, pp.159-163.

[5] B. V. Zabavsky, Diagonal reduction of matrices over rings, Mathematical Studies, Monograph Series, volume XVI, VNTL Publishers, Lviv, 2012.

[6] B. V. Zabavsky, Diagonal reduction of matrices over finite stable range rings, Mat. Stud., v.41, 2014, pp.101-108.

[7] W. Wm. McGovern, Neat ring, J. of Pure and Appl. Algebra, 205, 2006, pp.243-265.

[8] J. W. Brewer, W. J. Heinzer, On decomposing ideals in to product of comaximal ideals, Commun. Algebra, v.30, 2002, pp.5999-6010.

[9] M. D. Larsen, W. J. Lewis, T. S. Shores, Elementary divisor rings and finitely presented modules, Trans. Amer. Math. Soc., v.187, 1974, pp.231-248

[10] R. Gordon, J. C. Robson, Krull Dimension, Amer. Math. Soc., N.133, 1973, pp.1-78.

[11] B. V. Zabavsky, Fractionally regular Bezout rings, Matem. Stud., v.32, N.1, 2009, pp.76-80.

\section{CONTACT INFORMATION}

Oleh M. Romaniv Department of Mechanics and Mathematics, Ivan Franko National Univ., Lviv, Ukraine E-Mail(s): oromaniv@franko.lviv.ua Web-page(s): mmf.lnu.edu.ua/members/350

Bohdana

M. Kuznitska

Taras Ya. Hlova
Department of Building and Architecture, Lviv National Agrarian Univ., Dubliany, Ukraine E-Mail(s): kuznitska@ukr.net

Department of Engineering Mechanics, Hetman Petro Sahaidachnyi National Army Academy,

Lviv, Ukraine

E-Mail(s): hlovataras@ukr.net

Received by the editors: 20.06 .2018

and in final form 12.06.2020. 